\title{
A importância da monitoria acadêmica na ascensão à carreira docente
}

\author{
The importance of academic monitoring in the rise to teaching career \\ La importancia del seguimiento académico en el ascenso a la carrera docente
}

Recebido: 21/02/2021 | Revisado: 28/02/2021 | Aceito: 04/03/2021 | Publicado: 12/03/2021

\author{
Nataly Yuri Costa \\ ORCID: https://orcid.org/0000-0002-0476-9824 \\ Universidade do Estado do Pará, Brasil \\ E-mail: natalyyuricosta@gmail.com \\ Armando Sequeira Penela \\ ORCID: https://orcid.org/0000-0001-5644-635X \\ Universidade do Estado do Pará, Brasil \\ E-mail: armandopenela@hotmail.com.br \\ Laíze Rúbia Silva Corrêa \\ ORCID: https://orcid.org/0000-0002-9006-9161 \\ Universidade do Estado do Pará, Brasil \\ E-mail: laizerubia@gmail.com \\ Gabriela Xavier Pantoja \\ ORCID: https://orcid.org/0000-0002-0586-4487 \\ Universidade do Estado do Pará, Brasil \\ E-mail: gabyxavierpan@gmail.com \\ Samantha Stephany Prado Brazão \\ ORCID: https://orcid.org/0000-0002-3204-7896 \\ Universidade do Estado do Pará, Brasil \\ E-mail: samantha.brazao.ss@gmail.com \\ Paula Victória Tiribaxi Neves \\ ORCID: https://orcid.org/0000-0002-9115-2776 \\ Universidade do Estado do Pará, Brasil \\ E-mail: paulavictoria541@gmail.com \\ Nicole de Oliveira Araujo \\ ORCID: https://orcid.org/0000-0003-0403-1739 \\ Universidade do Estado do Pará, Brasil \\ E-mail: nickolearau@gmail.com \\ Leonardo Martins Silva \\ ORCID: https://orcid.org/0000-0001-6713-4968 \\ Universidade do Estado do Pará, Brasil \\ E-mail: leonardo.martins.nice@gmail.com \\ José Antonio Correa Gomes \\ ORCID: https://orcid.org/0000-0003-1596-3216 \\ Universidade do Estado do Pará, Brasil \\ E-mail: joseac1212@gmail.com \\ Sávio Felipe Dias Santos \\ ORCID: https://orcid.org/0000-0003-4066-5878 \\ Universidade do Estado do Pará, Brasil \\ E-mail: saaviofelipe@gmail.com \\ Breno Augusto Silva Duarte \\ ORCID: https://orcid.org/0000-0002-4003-7241 \\ Universidade do Estado do Pará, Brasil \\ E-mail: duartebreno30@gmail.com \\ João Gabriel Duarte de Lima \\ ORCID: https://orcid.org/0000-0002-8773-0334 \\ Universidade do Estado do Pará, Brasil \\ E-mail: freakgab@gmail.com
}

\begin{abstract}
Resumo
Objetivo: Relatar a experiência da monitoria acadêmica como colaboradora do desenvolvimento de habilidades da docência, segundo a perspectiva do acadêmico monitor. Método: Trata-se de um estudo descritivo, de abordagem qualitativa, do tipo relato de experiência, baseado nas vivências da monitoria acadêmica no componente curricular "Histologia Humana" ministrada em uma Universidade Pública, localizada em Belém do Pará, abordada no primeiro semestre no curso de graduação em Enfermagem, durante os anos de 2018 e 2019. Resultados: A experiência como discente-monitor favoreceu o desenvolvimento de habilidades docentes como ministrar aula, uso de metodologias ativas no processo de ensino aprendizado; comunicação; organização; compreender sobre as responsabilidades do
\end{abstract}


professor universitário; planejamento pessoal e profissional; liderança; trabalho em equipe e, principalmente, formação de vínculo e empatia com o monitorando, tornando um profissional crítico e autônomo. Conclusão: A monitoria acadêmica oportuniza ao discente-monitor aprendizados além do seu componente curricular, o qual exige atualização e estudo constante do aluno, em prol de um desempenho eficaz, junto ao docente, que supervisiona e orienta em suas funções, colaborando para o desenvolvimento de habilidades para um futuro magistério superior.

Palavras-chave: Docência; Aprendizagem; Monitoria.

\begin{abstract}
Objective: To report the experience of academic monitoring as a collaborator in the development of teaching skills, from the perspective of the academic monitor. Method: This is a descriptive study, with a qualitative approach, an experience report type, based on the experiences of academic monitoring in the curricular component " Human Histology " taught at a Public University, located in Belém do Pará, addressed in the first semester in the undergraduate course in Nursing, during the years 2018 and 2019. Results: The experience as student-monitor favored the development of teaching skills such as teaching classes, use of active methodologies in the teaching-learning process; Communication; organization; understand about the responsibilities of the university professor; personal and professional planning; leadership; teamwork and, mainly, bonding and empathy with the monitor, making a professional critical and autonomous. Conclusion: Academic monitoring gives the student-monitor the opportunity to learn in addition to its curricular component, which requires constant updating and study by the student, for the sake of an effective performance, with the teacher, who supervises and guides in their functions, collaborating for the development of skills for a future higher teaching profession.
\end{abstract}

Keywords: Teaching; Learning; Monitoring.

\title{
Resumen
}

Objetivo: Informar la experiencia de seguimiento académico como colaborador en el desarrollo de habilidades docentes, desde la perspectiva del monitor académico. Método: Se trata de un estudio descriptivo, con enfoque cualitativo, tipo relato de experiencia, basado en las experiencias de seguimiento académico en el componente curricular " Histología Humana " impartido en una Universidad Pública, ubicada en Belém do Pará, abordada en el primer semestre de la carrera de Licenciatura en Enfermería, durante los años 2018 y 2019. Resultados: La experiencia como estudiante-monitor favoreció el desarrollo de habilidades docentes como la impartición de clases, uso de metodologías activas en el proceso de enseñanza-aprendizaje; Comunicación; organización; comprender las responsabilidades del profesor universitario; planificación personal y profesional; liderazgo; trabajo en equipo y, sobre todo, vinculación y empatía con el monitor, convirtiéndose en un profesional crítico y autónomo. Conclusión: El seguimiento académico brinda al alumno-monitor oportunidades de aprendizaje más allá de su componente curricular, lo que requiere una constante actualización y estudio por parte del alumno, a fin de desempeñarse eficazmente, junto con el docente, quien supervisa y orienta en sus funciones, colaborando para la desarrollo de habilidades para una futura profesión docente superior.

Palabras clave: Enseñanza; Aprendizaje; Seguimiento.

\section{Introdução}

A monitoria acadêmica enquanto um serviço de apoio pedagógico oportuniza ao discente-monitor aprofundar conhecimentos teóricos e práticos, habilidades e técnicas, em conjunto com o docente (De freitas; Alves, 2019). Viabiliza o fortalecimento do processo ensino-aprendizagem não somente dos alunos monitorados, como também do discente monitor, visto que além de esclarecer dúvidas sobre determinada disciplina, preenchendo as lacunas de conhecimento, possui vivências do significado do "ser docente universitário", compreendendo a importância do planejamento pedagógico, do vínculo e confiança com os alunos, comunicação, organização e responsabilidade sobre o componente curricular e o entendimento deste pelos estudantes (Fontes et al., 2019).

Com objetivo de aperfeiçoar ensino e desenvolver habilidades técnico-didáticas na formação superior a Lei ${ }^{\circ}$ 5.540/1968 regulamentou a monitoria acadêmica (Brasil, 1968), sendo validada, posteriormente, pela Lei nº 9.394/1996, que estabelece as diretrizes e bases da educação nacional (Brasil, 1996), no propósito de ofertar e fortalecer o tripé ensino, pesquisa e extensão, analisando e desenvolvendo as aptidões dos estudantes.

Nesse cenário, em 2015 foi aprovada a resolução 2.808/2015, do Conselho Superior Universitário (Consun), que normatiza o programa de monitoria na conjuntura da Universidade do Estado do Pará (UEPA), contemplada pelo edital da Próreitoria de Graduação de Ciências Biológicas e da Saúde da Universidade do Estado do Pará (UEPA). O programa de 
monitoria acadêmica tem como objetivo ampliar a participação do aluno de graduação na vida acadêmica; complementar a formação acadêmica do discente monitor; possibilitar o desenvolvimento de habilidades de caráter pedagógico no discentemonitor; contribuir para a redução de problemas de repetência e evasão; contribuir para o aprimoramento do ensino através do desenvolvimento de novas práticas e experiências pedagógicas no processo ensino-aprendizagem - avaliação, voltadas para a melhoria do ensino do componente curricular objeto da monitoria.

Com isso, é essencial fortalecer o processo de ensino- aprendizagem proporcionando ao discente-monitor a oportunidade de visualizar as funções e desempenho do docente, compreendendo as metodologias de ensino, fundamentando e fortalecendo seus conhecimentos sobre o componente curricular; desenvolvendo funções da monitoria, desde que esteja em consonância com o projeto político pedagógico do curso (Fernandesa et al., 2015).

Na pesquisa de De Andrade et al. (2018) com docentes e discentes foi discutida a monitoria acadêmica como um instrumento de troca de saberes, experiências e aprendizado mútuo, seja entre monitor e o docente, seja entre monitor e os monitorados. Trata-se de uma relação dinâmica, contínua e participativa, sendo o monitor um aluno com conhecimentos mais aprofundados e habilidoso, por já ter passado pela disciplina, que facilita o processo de ensino-aprendizagem a partir de metodologias ativas, contribuindo em conjunto com o docente-orientador um maior aproveitamento da disciplina, esclarecendo dúvidas e estabelecendo uma relação de confiança com os discentes, desenvolvendo habilidades que fomentam a ascensão ao magistério superior.

A monitoria acadêmica instiga potenciais habilidades de um professor universitário, essencial para carreira magisterial, estimulando o discente-monitor a expandir seu próprio repertório didático. A partir das suas contribuições ao docente, o monitor desenvolve seu perfil individual enquanto educador, abrangendo diversas modalidades de ensino, além de desenvolver a comunicação; expressão corporal; planejamento pessoal e profissional; responsabilidade; liderança; trabalho em equipe e, principalmente, formação de vínculo e empatia com o educando, tornando um profissional crítico e autônomo, fundamental no êxito enquanto docente (Botelho et al., 2019). O objetivo desse artigo é relatar a experiência da monitoria acadêmica como colaboradora do desenvolvimento de habilidades da docência, segundo a perspectiva do acadêmico monitor.

\section{Metodologia}

Trata-se de um estudo descritivo, com abordagem qualitativa, que tem o propósito de observar, descrever e documentar uma realidade, do tipo relato de experiência (Polit; Beck, 2018; Pereira et al., 2018), baseado na vivência da monitoria acadêmica, durante o período de outubro de 2018 a dezembro de 2019, após a aprovação no processo seletivo de ${ }^{\circ}$ 52 - 2018 para o componente curricular "Histologia Humana". Esta disciplina é ministrada no $1^{\circ}$ semestre do curso de Graduação em Enfermagem pela Universidade do Estado do Pará (UEPA), sendo parte do Departamento de Morfologia e Ciências Fisiológicas (DMCF), exigindo a carga horária de 15 horas semanais, divididas em 2 aulas, durante 10 meses, distribuídas em atividades desempenhadas pelo monitor.

As estratégias e recursos de ensino utilizados nas aulas teóricas incluem aulas expositivas dialogadas, dinâmicas, vídeos, discussão de artigos científicos e seminários. Nas atividades práticas, os acadêmicos visualizavam no microscópio a lâmina referente ao conteúdo ministrado, além de resolver exercícios de fixação, a fim de um melhor aprendizado. As principais estratégias de ensino utilizadas foram dinâmicas de grupo, seminários e jogos, promovendo um ambiente descontraído e educativo.

O monitor da disciplina cumpriu carga horária semanal de quinze horas, abrangendo atividades de levantamento bibliográfico de temas pertinentes à disciplina, acompanhamento dos acadêmicos nas atividades práticas e orientação dos alunos no planejamento das ações a serem implementadas. Todas as atividades do monitor foram desenvolvidas sob supervisão direta do docente. 


\section{Resultados e Discussão}

Dentre as funções do monitor estava acompanhar os alunos durante as aulas teóricas e práticas. As aulas teóricas aconteciam em sala de aula, e o monitor colaborava com a retirada de dúvidas dos alunos em conjunto com o docente acerca do conteúdo ministrado. As aulas práticas ocorriam no laboratório logo após a aula teórica, sendo um momento em que o monitor tinha autonomia de ajudar os alunos a manusear o microscópio, visualizar na lâmina e esclarecer o conteúdo teórico vinculando à prática. A cada aula ministrada era repassado exercícios de fixação feita pelo monitor, e questões de processos seletivos para os alunos, a fim do melhor aprendizado.

A cada conteúdo finalizado, o monitor enviava um material para os alunos em power point resumido sobre os assuntos predominantes nas avaliações, e quando próximo do período das provas era realizado revisões teóricas e práticas, fortalecendo uma relação livre e confortável entre os discentes e o monitor, além de amenizar as lacunas sobre o conteúdo, contribuindo para o maior aproveitamento da disciplina.

Uma estratégia de ensino prática e eficaz utilizada foi o uso de Tecnologias Educacionais Digitais (TED), na forma de jogos educativos virtuais, como a plataforma Kahoot, o qual o aluno jogava um quiz game a partir de perguntas formuladas pelo monitor sobre a disciplina Histologia Humana, e os alunos respondiam convertendo em pontos e premiações (Sande; Sande, 2018). A partir das dúvidas que iam surgindo, o monitor sanava ao máximo em sala de aula, ou por meio de plantões de dúvidas via ferramentas digitais, como aplicativo de mensagens instantâneas.

Além disso, o monitor acompanhava os alunos nas pesquisas de campo, auxiliando na visualização das problemáticas e precariedades locais, elencando potenciais discussões que poderiam ser amenizados com intervenções futuras, sendo exigido ao final do semestre um trabalho escrito, documentando os resultados da pesquisa, o qual o monitor orientava e corrigia, em conjunto com o docente, dentre outras atividades realizada pelo discente-monitor descritas no Quadro 1.

Quadro 1 - Atividades desenvolvidas durante a monitoria de "Histologia Humana"

\begin{tabular}{|l|}
\hline 1. Elaboração de materiais teóricos e exercícios de fixação, com base no referencial bibliográfico \\
\hline 2. Grupos de estudos em prol da retirada de dúvidas \\
\hline 3. Acompanhamento nas aulas teóricas e práticas, conduzindo os alunos no uso do microscópio, \\
localização de células e aplicação do conteúdo ministrado à pratica \\
\hline 4. Aplicação das avaliações bimestrais \\
\hline $\begin{array}{l}\text { 5. Apresentação de trabalhos referentes a monitoria em eventos acadêmicos, como congressos e } \\
\text { simpósios }\end{array}$ \\
\hline 6. Planejamento semestral em conjunto com o docente \\
\hline 7. Colaboração na leitura e correção de trabalhos científicos dos alunos \\
\hline
\end{tabular}

Fonte: Autoria Própria.

\section{Atuação do monitor e o desenvolvimento das habilidades docente}

A presença do discente-monitor em sala de aula facilitou o processo ensino aprendizagem, partindo do seu conhecimento prévio sobre a disciplina, intensificando a relação professor-aluno- instituição. Nesse sentido, o monitor torna-se autônomo em utilizar diversas ferramentas de aprendizagem, podendo ser criativo e dinâmico, superando a monotonia didática profissional, a fim do preenchimento de lacunas sobre o conteúdo. Há um maior aproveitamento da disciplina, permitindo uma relação próxima com o aluno, identificando seu perfil e dificuldades, uma vez que pode haver receios quanto aos questionamentos ao docente, consolidando, assim, um educador, aspirante à atuação profissional docente (Fernandesb et al., 2015). 
Durante o desempenho em sala de aula, foi consolidado uma relação próxima entre o docente e o monitor, pois era discutido o plano de ensino pedagógico semestral; quais conteúdos a serem ministrados; atividades a serem desenvolvidas com os alunos e critérios avaliativos. Assim, era evidenciada as diversas atribuições ao professor universitário, sendo vivências práticas que fomentam no discente-monitor o desenvolvimento individual de um educador crítico, ciente de suas responsabilidades e deveres.

No estudo de Mesquita et al. (2019) foi relatado que a monitoria de multidisciplinar de anatomia e histologia foi grande valia ao monitor, agregando valores e significados a formação profissional docente, desde de o âmbito acadêmico. Tomada de decisão, comando de voz, trabalho em equipe, escuta ativa e o uso de metodologias ativas no processo ensinoaprendizagem foram destacados como habilidades potencializadas na monitoria acadêmica, essenciais para a ascensão a carreira magisterial.

A docência caracteriza-se por uma troca de experiências e saberes, os quais o professor enquanto educador deve dominar metodologias de ensino, para que o aluno absorva e internalize o conteúdo. Assim, o uso das novas tecnologias de informação no processo ensino- aprendizagem ganham notoriedade, facilitando a comunicação interpessoal, acesso às atividades via online, estreitando a relação aluno-monitor (Félix et al., 2018). Dessa forma, a monitoria acadêmica como um espaço de liberdade, criatividade e autonomia, o monitor possui um arsenal de ideias que permeiam um processo de ensino contínuo e eficiente, se apropriando de estratégias inovadoras, como a gamificação (Sande; Sande, 2018).

Nos cursos da saúde, historicamente, é comum o ensino baseado em modelos tradicionais, bancários, em que o professor é o centro do processo formativo e o aluno é um mero receptáculo de informações. Assim, é importante que seja impulsionado o desenvolvimento de novas habilidades para um futuro docente, indo além de uma metodologia monótona, superando a reprodução de padrões cartesianos, colocando em prática metodologias ativas que instiguem a reflexão e pensamento crítico (Moreira, 2014).

Dentre as habilidades desenvolvidas durante a monitoria, a comunicação; comando de voz; falar em público; saber se expressar e explicar de forma assertiva e clara foram destacadas, competências fundamentais para um professor universitário. No estudo de Cacciari et al. (2017) foi realizado uma pesquisa sobre as principais características/virtudes de um bom professor, dentre elas foram destacadas "Habilidade de comunicação" e "Didático", essenciais na prática docente. Assim, durante a monitoria acadêmica foi percebido um cenário fértil para o desenvolvimento de tais características/virtudes, visto que é dever do monitor revisar conteúdos, tirar dúvidas, por meio de estratégias didáticas e assertivas, que reforcem o aprendizado e contribua para a formação acadêmica dos discentes.

O monitor é um eterno aprendiz, que precisa estar em constante estudo, atualização do conhecimento e aperfeiçoamento da prática, sendo assim, percebe-se a necessidade do aprendizado contínuo, para um bom desempenho no meio acadêmico, melhorando suas aptidões, seja como discente ou um futuro docente. O exercício da monitoria é um subsídio à prática docente, já que o monitor não somente complementa seus conhecimentos, como também adquire vivências da rotina de um professor universitário, capacidade de interação, avaliando a postura diante de determinadas situações, absorvendo fundamentos teóricos e práticos, e possuindo vivências que permitam novas maneiras de pensar o saber e o fazer (Branco Junior et al., 2018).

\section{Contribuição da monitoria na formação profissional}

As vivências da monitoria acadêmica possibilitaram visualizar a importância de semear, desde de a graduação, habilidades e características que moldam um profissional qualificado, humanizado, ciente do seu impacto como educador na transformação da realidade. Ao desempenhar suas atividades, o discente- monitor pode rever conteúdos e reinterpretá-los, o que permitiu um aprofundamento na teoria, consolidação de conteúdo, estabelecendo uma relação próxima com os discentes, 
percebendo suas dificuldades. Assim, é fundamental que ao concluir a graduação, independente de área, seja formado um indivíduo capacitado, empático nas relações interpessoais, consciente do seu papel como cidadão, que desempenhe suas funções com compromisso, respeito, pontualidade, sendo humanizado e crítico na sua conduta. (Roman et al., 2017).

No estudo de Burgos et al (2019) com monitores do curso de graduação em enfermagem foi evidenciado a monitoria acadêmica como fundamental no desenvolvimento teórico-prático do conteúdo ministrado, compreendendo o exercício profissional na prestação de cuidados, trabalho em equipe, habilidade de se comunicar com clareza, realizando educação em saúde eficaz e efetiva; gestão e gerenciamento dos serviços de saúde, criando segurança e precisão, desenvolvendo habilidades durante as práticas clínicas, competências éticas, políticas, importantes para o crescimento pessoal e profissional.

Durante a formação universitária é fundamental que os discentes desenvolvam a capacidade de reflexão, de discussão, e fortalecimento de pensamentos críticos e pertinentes sobre sua realidade e atuação em sociedade, tornando profissionais comprometidos, aptos a trabalharem em equipe. Ensinar, na concepção de Paulo Freire trata-se de utilizar ferramentas problematizadoras que instiguem um aprendizado ativo, emancipador, que promova questionamentos, que seja baseado na reciprocidade entre o educador e educando, e forme cidadãos com senso de responsabilidade social (Chiarella et al., 2015).

Em sua obra Pedagogia da autonomia, Freire afirma que na formação docente, futuro magistério superior, é necessário que, desde a academia, desenvolva o ensino metódico, que estimule o raciocínio crítico, atitudes éticas, promovendo a autonomia e a capacidade de criar juízo sobre as circunstâncias o qual se insere (Freire, 2004). O despreparo acerca das habilidades de ensino contribui para um processo de ensino aprendizagem superficial, monótono, baseado em modelos bancários, os quais são ultrapassados e cria lacunas de conhecimento. Assim, a monitoria acadêmica enquanto experiência no processo de ensino baseado em metodologias ativas possibilita que o discente valorize um aprendizado com bases éticas, humanísticas, desenvolvendo habilidades que vão além do conteúdo ministrado, como comunicação, empoderamento, liderança, confiança entre o discente-monitor e o aluno, empatia responsabilidade e compromisso para com suas funções (De freitas; Alves, 2019).

\section{Considerações Finais}

Este trabalho contribuiu para o aprendizado sobre a importância da monitoria acadêmica na ascensão ao magistério superior na perspectiva do discente- monitor, não somente sobre a atuação do docente universitário na sua conduta frente as suas responsabilidades, como também o desenvolvimento de habilidades que facilitam o processo de ensino- aprendizagem, como organização; comunicação; expressão corporal; planejamento pessoal e profissional; liderança; trabalho em equipe; tomada de decisão; formação de vínculo e empatia com o educando, com o objetivo de formar um profissional com capacidade de julgar, senso crítico e autonomia.

A monitoria acadêmica é tida como uma oportunidade de o discente desenvolver competências características do "ser docente" dentro da graduação, compreendendo que ensinar não é restrito ao repasse de informações, trata-se de refletir sobre o que é ensinado, estimular debates sobre o conteúdo, formulando hipóteses, ouvindo diversas opiniões, sanando dúvidas e, principalmente, estabelecendo uma relação estreita com os monitorandos, criando vínculos e confiança. É fundamental que o processo de ensino-aprendizagem seja baseado, sobretudo, na formação de caráter, conscientização sobre seu papel como cidadão, humanizado e empático na sua conduta, compromissado com seus deveres, fatores estes instigados na monitoria acadêmica.

Em síntese, é necessário que haja mais publicações sobre esse tema, uma vez que fortalece discussões o papel do discente-monitor, amplia a visão do processo ensino- aprendizagem com base em metodologias ativas, permite maior 
visibilidade da sua importância e expansão dos programas monitoria pelas instituições de ensino superior e, principalmente, beneficia o aluno, quando bolsista, no auxilio de custos, além de colaborar com experiência e conhecimento.

\section{Referências}

Brasil. Ministério da Educação. Lei no 9.394, de 20 de dezembro de 1996. Lei de Diretrizes e Bases da Educação Nacional [internet]. Diário Oficial da União, Brasília, 23 de dez, 1996.

Brasil. Senado Federal, Lei Federal no 5540, de 28 de novembro de 1968. Lei de Diretrizes e Bases da Educação Nacional [internet]. Diário Oficial da União, Brasília, 29 de novembro de 1968 .

Botelho, L. V., et al. (2019). Monitoria acadêmica e formação profissional em saúde: uma revisão integrativa, ABCS Health Sciences, 44 (1), 67-74.

Branco Junior, A. G., et al. (2018). Monitores no processo de ensino aprendizagem: avaliação da tríade envolvida, Revista Multidisciplinar em Educação, 5 (10), 149-164.

Burgos, C. N., et al. (2019). Monitoria acadêmica na percepção de acadêmicos de enfermagem. Revista de Enfermagem da UFSM, 9 (e37), 1-14.

Cacciari, M. B., et al. (2017). Percepções de professores universitários brasileiros sobre as virtudes mais valorizadas no exercício da docência, Psicologia Escolar e Educacional, São Paulo, 21 (2), 313-322.

De Andrade, E. G. R., et al. (2018). Contribuição da monitoria acadêmica para o processo ensino-aprendizagem na graduação em enfermagem, Revista Brasileira de Enfermagem REBEn, 71 (2), 1690- 1698.

De Freitas, F. A. M, Alves, M. I. A. (2020). Construindo uma identidade acadêmica: reflexão acerca da monitoria no IEAA/UFAM. RECH- Revista Ensino de Ciências e Humanidades - Cidadania, Diversidade e Bem Estar, 4 (1), 281-299.

Félix, D. F et al. (2018). Monitoria em anatomia: a percepção dos acadêmicos de medicina, Archives Health Sciences, 25 (3), $53-55$.

Fernandesa, N. C., et al. (2015). Monitoria acadêmica e o cuidado da pessoa com Estomia: relato de experiência, Revista Mineira de Enfermagem REME, 19 (2), 238-241.

Fernandesb, M. A., et al. (2015). Monitoria no ensino das paixões: acolhimento ao aluno no primeiro contato com a psicopatologia, Revista de Psicanálise Analytica, 4 (6), 138-150.

Freire, P. (2004). Pedagogia da autonomia: saberes necessários a prática educativa: Paz e Terra.

Fontes, F. L. L. et al. (2019). Contribuições da monitoria acadêmica em Centro Cirúrgico para o processo de ensino-aprendizagem: benefícios ao monitor e ao ensino. Revista Eletrônica Acervo Saúde, São Paulo, 27 (e901), 1-6.

Mesquita, G. N., et al. (2019). Métodos de ensino integrados em monitoria de anatomia e Histologia: um relato de experiência, Revista Eletrônica Acervo em Saúde, 30 (e1370), $1-5$

Moreira, D. L. (2014). Dificuldade de aprendizagem: um conceito oriundo da educação bancária, 2014.81 f (Mestrado em Psicologia) - Centro Universitário de Brasília-UniCEUB, Brasília.

Pereira, A.S. et al. (2018). Metodologia da Pesquisa Científica. UFSM.

Polit, D., \& Beck, C. (2018). Fundamentos de pesquisa em enfermagem: avaliação de evidências para a prática da enfermagem (9.ed.). Artmed.

Roman, C., et al. (2017). Metodologias ativas de ensino-aprendizagem no processo de ensino em saúde no Brasil: uma revisão narrativa, Clinical \& Biomedical Research, 37 (4), 349- 357.

Sande, D \& Sande, D. (2018). Uso do kahoot como ferramenta de avaliação e ensino-aprendizagem no ensino de microbiologia industrial, HOLOS, 1, 170180 .

Universidade Do Estado Do Pará. Conselho Universitário. Resolução n ${ }^{o}$ 2.808/15 - CONSUN, 18 de março de 2015 ; 2015.15 p. Fixa normas complementares para execução do programa de monitoria no âmbito da Universidade do Estado do Pará. 